\title{
Clinical Patterns and Renal Survival of Nephrotic Syndrome in Childhood: A Single-Center Study (1980-2006)
}

\author{
Svitlana Fomina ${ }^{*}$, , Tatjana Pavlenko ${ }^{*}, 2$, Erling Englund ${ }^{3}$ and Ingretta Bagdasarova ${ }^{1}$ \\ ${ }^{I}$ Department of Pediatrics, State Institution «Institute of Nephrology of the Academy of Medical Science of Ukraine», \\ Kyiv, Ukraine \\ ${ }^{2}$ Department of Statistics, Stockholm University, Stockholm, Sweden \\ ${ }^{3}$ Västernorrland County Council, Research and Development Centre, Sundsvall, Sweden
}

\begin{abstract}
To investigate changes in the diagnostic patterns, disease profiles courses and therapeutic strategies for severe forms of childhood nephrotic syndrome (NS), the clinical features of 1349 children treated during two consecutive time periods, 1980-2000 ( $\mathrm{n}=1$ 162) and 2001-2006 $(\mathrm{n}=187)$, were retrospectively reviewed. The significant increase in initial renal impairment, NS with hypertension, and NS with hypertension and hematuria was observed $(27.7 \%$ vs $51.3 \%, 1.0 \%$ vs $5.3 \%$ and $16.4 \%$ vs $21.9 \%$, respectively). The rate of both secondary steroid resistance (SR) and Focal Segmental Glomerulosclerosis increased significantly, $(1.8 \%$ vs $5.6 \%, \mathrm{p}=0.032$, and $14.9 \%$ vs $29.0 \%, \mathrm{p}=0.034$, respectively). The initial renal insufficiency and hypertension were highly predictive of the development of stage 3 of chronic kidney disease (CKD3) among SR patients in a multivariate Cox regression $(p=0.001)$ for years 1980-2000. A higher hazard of CKD3 in male SR patients from three to six years old was observed in 2001-2006. Kaplan-Meier survival curves revealed a shift in the cumulative probability of CKD3, indicating a slower decline of the renal function for SR NS in the years 2001-2006 $(\mathrm{p}=0.008)$ : the estimated five-year CKD3 risk was $39.7 \%$ vs $27.7 \%$. Achievements in inducing remission and retarding the development of CKD3 in combination with increased severity of NS indicate the effectiveness of domestic strategies of NS management.
\end{abstract}

Keywords: Nephrotic syndrome, child, pathology, outcome, renal survival.

\section{INTRODUCTION}

The nephrotic syndrome (NS) is a relatively rare but severe form of glomerulopathy in children. The estimated global annual incidence rate of NS is $1.9-2.6$ cases per 100000 children [1-6]. In general, nearly $80 \%$ cases of childhood NS are steroid sensitive (SSNS) and respond well to steroid treatment with favorable outcome [1, 7-9]. However, steroid resistance (SRNS) has been associated with adverse outcome in terms of potential development of stage 3 of chronic kidney decease (CKD3) [2, 10].

The disparity between the clinical presentation, and the observed histopathological patterns, is the most important impediment in the prediction of disease outcomes and the development of safe and effective strategies in the management of childhood NS.

The aim of the present study was to investigate and compare the clinical, epidemiological, histological patterns and treatment response among the Ukrainian children with NS during two consecutive time periods (1980-2000 and 2001-2006) besides the assessment of prognostic factors that modify the risk of development of End Stage Renal Disease (ESRD) in this part of population.

*Address correspondence to these authors at the (SF) State Institution «Institute of Nephrology of the Academy of Medical Science of Ukraine», Department of Pediatrics, 17b Degtyarivska str, Kiev, Ukraine; Tel/Fax: +38044 2853644; E-mail: sfomina@meta.ua, and (TP) Stockholm University, Department of Statistics, S-106 91 Stockholm, Sweden; Tel: +46 8 162578; Fax: +46 8 167511; E-mail: Tatjana.Pavlenko@stat.su.se

\section{MATERIALS AND METHODOLOGY}

In the present longitudinal retrospective study a cohort of 1349 children with uniform ethnic background having NS as their primary diagnosis, were followed up in the referral clinic between January 1980 and May 2006. All patients manifested the most severe courses of NS characterized by frequent relapses or none response to initial steroid treatment in the local clinics.

To capture changing trends of clinical courses, responsiveness to the treatment and outcomes in the cohort under study, we specified two time intervals, from 1980 to 2000 and from 2001 to 2006. These intervals corresponded to the principally different strategies in management of childhood NS. The treatment approach accepted during the latter time period was relatively close to international protocols and was characterized by reduced cumulative doses of immunosuppressant in combination with pulse therapy. The treatment methods during the former time period (1980-2000) were essentially based on the domestic protocols with prolonged steroid and cytotoxic therapy. Both strategies, however, rely on the more intensive courses of immunosuppressant which are in contrast to the international recommendations.

Children with age of NS onset $<1.5$ years, follow-up $<3$ years and those with secondary NS, were excluded from the study. The data reviewed were: age of onset, duration of disease, the relapse rate before the beginning of the treatment course in the clinic, renal histology, and response to steroid therapy, serial blood pressure measurements, and the 
outcomes. We also examined the biochemical data before and at regular intervals after the initiation of the treatment, including serum creatinine, creatinine clearance, serum albumin, cholesterol, urinalysis, and 24-h urinary protein excretion. The patients were followed up once a week in the first three months, every three months in the first year, and every six months thereafter.

Cohort of children was divided into five groups: from 1.5 to $<3$ years (1), from three to $<6$ years (2), from six to $<9$ years (3), from nine to $<12$ years (4) and, from twelve to 17 years (5).

The Ethics Committee of the Institute of Nephrology of the AMS of Ukraine approved of the study.

\section{Definitions}

A "classic" NS was defined by the presence nephroticrange proteinuria (urinary protein level $>3 \mathrm{~g} / 24$ hours, in the youngest age group $-1 \mathrm{~g} / \mathrm{m}^{2}$ ), serum albumin $<25 \mathrm{~g} / \mathrm{L}$, serum cholesterol $>6.6 \mathrm{mmol} / \mathrm{L}$, and edema $[11,12]$.

Hematuria was defined as $>5 \times 10^{6}$ red blood cells $/ \mathrm{L}$ in uncentrifuged urine [12].

Hypertension was defined as blood pressure (systolic and/or diastolic) $>95^{\text {th }}$ percentile for age, gender, height percentile.

Initial renal insufficiency was defined as an increase of the serum creatinine level $(100-460 \mu \mathrm{mol} / \mathrm{l})$ in the initial observation of less then three months. It was accompanied by a decrease of the glomerular filtration rate according to the Schwartz formula [13, 14].

SS NS was defined as a complete remission after six weeks of prednisolone (PRED) with a dose $1.5-2 \mathrm{mg} / \mathrm{kg} /$ day. SR NS was defined as partial remission or no remission. Patients who responded to PRED therapy during the initial episode, but failed to respond in a subsequent relapse, were diagnosed with secondary SR $[11,12]$.

Treatment response was classified as [11]:

- complete remission (total resolution of proteinuria and edema, serum albumin $>30 \mathrm{~g} / \mathrm{L}$, serum cholesterol $<6.6 \mathrm{mmol} / \mathrm{L}$ );

- partial remission (loss of edema, reduction to nonnephrotic levels proteinuria $<2 \mathrm{~g} /$ day, decrease of changes in serum);

- none remission (the same NS status as at the beginning of the observation in the clinic with the persistence of nephrotic-range proteinuria, normal renal function - the glomerular filtration rate and serum creatinine level in age-related normal levels).

CKD3 was defined as no remission with a persistent decrease of the glomerular filtration rate $<60 \mathrm{ml} / \mathrm{min} / 1.73 \mathrm{~m}^{2}$ (not less than $30 \mathrm{ml} / \mathrm{min} / 1.73 \mathrm{~m}^{2}$ ) for three months or longer [12].

Relapse was defined as the recurrence of nephrotic range proteinuria ( $>3 \mathrm{~g} /$ day) with serum abnormality (albumin $<25$ $\mathrm{g} / \mathrm{L}$, cholesterol $>6.6 \mathrm{mmol} / \mathrm{L}$ ) after the urine had been protein free for at least four weeks [12].

\section{Treatment Protocol}

Our patients were treated initially for six weeks with 1.5 $2.0 \mathrm{mg} / \mathrm{kg}$ body weight for PRED up to a maximum of 60 $\mathrm{mg} /$ day, divided into two or three doses (Fig. 1) [11].

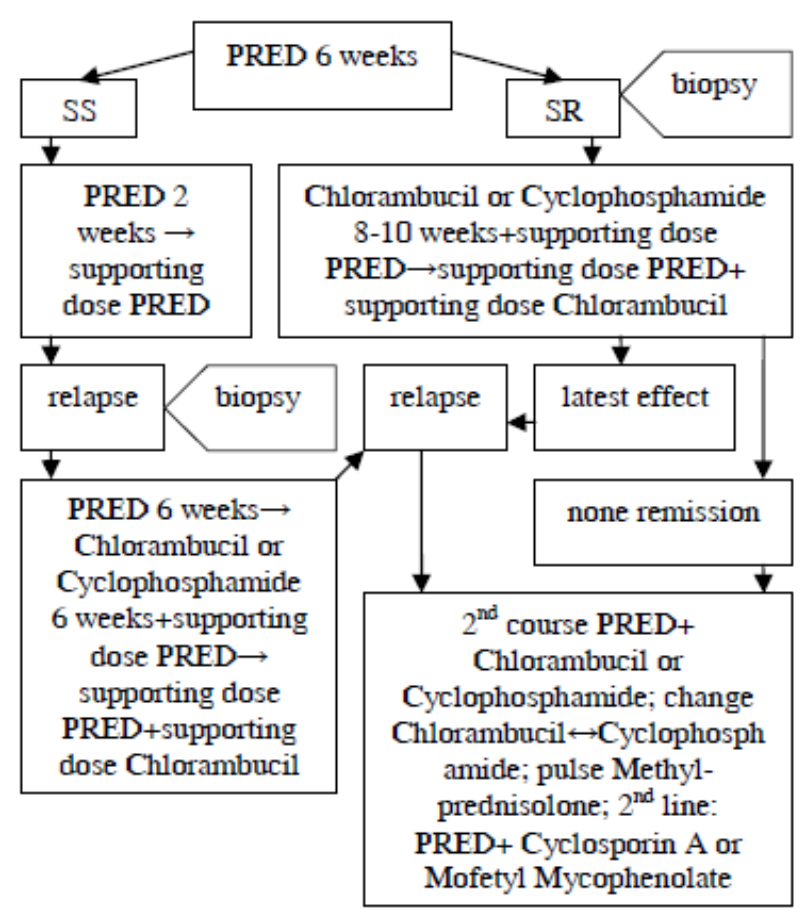

Fig. (1). Flow chart of treatment algorithm used in children with NS: PRED and immunosuppressive agents (NS nephrotic syndrome, $S S$ steroid sensitive, $S R$ steroid resistance, PRED prednisolone).

In the case of SS, the same dose of PRED was prolonged up to eight weeks, after which the steroid agent was decreased by $5 \mathrm{mg} /$ day to the supporting dose $(30-50 \%$ of initial dose). The supporting dose was given for 6-9 months, either every second day or intermittent mode (three times a week) with tapered by $2.5 \mathrm{mg}$ every 6-8 weeks till $2.5-5 \mathrm{mg}$.

Since 2000, the protocol with pulse Methylprednisolone was used. The dose of Methylprednisolone was 20-30 $\mathrm{mg} / \mathrm{kg} /$ day $(\leq 1 \mathrm{~g})$ every other day, three times in total. Then, given that no side effects have been observed, it was once a week, two to four times in total [15].

An immunosuppressive agent was prescribed when remission could not be achieved in six weeks of PRED or when relapse occurred. The first line steroid-sparing agent was either Chlorambucil or Cyclophosphamide prescribed for 8-10 weeks. Chlorambucil was received in dose 0.15-0.2 $\mathrm{mg} / \mathrm{kg} /$ day orally (cumulative dose 8.5-10 $\mathrm{mg} / \mathrm{kg}$ ). Cyclophosphamide was initiated at a pulse dose intravenously (I.V.) of $10-25 \mathrm{mg} / \mathrm{kg}$ once in three weeks ( $\leq 1$ g) three times or intramuscular (I.M.) every third day in doses 6-9 mg/kg/injection (cumulative dose $120-150 \mathrm{mg} / \mathrm{kg}$ ). After the maximum dose of immunosuppressive agent the treatment was switched to the supporting dose of Chlorambucil $(0.1 \mathrm{mg} / \mathrm{kg} / \mathrm{d})$ in all cases for $6-12$ months 
period (cumulative dose 20-35 $\mathrm{mg} / \mathrm{kg}$ ) together with supporting dose of PRED.

\section{Renal Biopsy}

25 renal biopsies, performed in the years 2004-2006, as well as the 114 biopsies that performed before the year 2004 and might be subsequently reviewed, were included in the study. There were done blindly (1980-1998) or under ultrasound guidance (1998-2006). All the biopsies corresponded to SR NS. $27.3 \%$ (38) of biopsies were performed no later than six months after the disease onset, and 30.2\% (42) of all biopsied patients had at least three years of NS history. No subsequent biopsy was performed.

All the renal biopsies were examined by light microscopy. Immunofluorescence was introduced only in the year 2004. The electron microscopy was done since the year 2001 when discrepancies between the clinical patterns and light microscopy were observed or in a case when the secondary NS was suspected.

All histological data were reanalyzed by one renal pathologist in the year 2008 who was blind to patients' clinical courses and outcomes. He reached the conclusion coincided with the previous diagnosis.

\section{STATISTICAL ANALYSIS}

The statistical analyses were performed using STATISTICA 8.0 for Windows Version (Statsoft, Inc. 19842008) and SPSS 16.0 (SPSS Inc. 1989-2007). Student t-test, Mann-Whitney test and the Fisher exact test were used to analyze and compare the variables in question. Time dependent risk of development of CKD3 among two groups was estimated by Kaplan Meier survival method and statistical comparison of survival rates was performed using log-rank test [16]. The Cox proportional hazards regression technique was applied to explore the simultaneous effects of several explanatory variables on the risk of development CKD3 [16, 17].

\section{RESULTS}

The present study included 1349 children (788 male and 561 female) with mean age of 6.7 ( \pm 3.98 , range $1-16)$ years and mean disease duration of $12( \pm 15.1$, range $1-168)$ months. The characteristics of children according to the different time period are shown in Table $\mathbf{1}$.

Table 1. Demographics of Patients at Presentation

\begin{tabular}{|l|c|c|}
\hline \multirow{2}{*}{} & \multicolumn{2}{|c|}{ Time Period } \\
\cline { 2 - 3 } & $\mathbf{1 9 8 0 - 2 0 0 0}$ & $\mathbf{2 0 0 1 - 2 0 0 6}$ \\
\hline \hline No. of patients & 1162 & 187 \\
\hline Gender ratio (male/female) & 1.4 & 1.3 \\
\hline Age of disease onset, years (mean \pm SD) & $6.7 \pm 3.9$ & $6.8 \pm 47$ \\
\hline $\begin{array}{l}\text { Duration before the beginning of the } \\
\text { treatment in clinic, months (mean } \pm \mathrm{SD})\end{array}$ & $11 \pm 13$ & $16 \pm 24$ \\
\hline Follow-up, months (mean $\pm \mathrm{SD})$ & $65 \pm 44$ & $30 \pm 17$ \\
\hline
\end{tabular}

No significant difference was observed for average age of onset of disease between the two periods. However, the mean duration of NS before observation in our clinic was significantly higher in 2001-2006 ( $\mathrm{p}=0.001)$.

Changes of the NS age distribution were revealed between the two time intervals (1980-2000 and 2001-2006). A significant decrease of the proportion of the patients in the age groups traditionally sensitive to PRED (2 and 3) was observed in combination with an increase of NS in the youngest and oldest ones (1 age group: from $11.2 \%$ (130) to $14.4 \%$ (27), $\mathrm{p}=0.041 ; 2$ group: from $27.3 \%$ (317) to $23.0 \%$ (43), $\mathrm{p}=0.034 ; 3$ group: from $23.6 \%$ (275) to $14.4 \%$ (27), $\mathrm{p}=0.001 ; 4$ group: $17.6 \%$ (204) to $20.3 \%$ (38), $\mathrm{p}=0.052 ; 5$ group: from $20.3 \%$ (236) to $27.9 \%(52), \mathrm{p}=0.006)$.

Significant differences in the clinical presentations of NS were observed among the two specified time periods. During the years 1980-2000, "classic" NS and NS with hematuria were diagnosed in 59.4\% (691) and 23.2\% (270) cases, respectively. NS with isolated hypertension and NS with combined hematuria and hypertension were discovered in $1.0 \%$ (11) and $16.4 \%$ (190), respectively. After the year 2000 a significant increase in the proportion of NS patients with hypertension and those with accompanied hematuria were found (5.3\% (10), $\mathrm{p}<0.001$, and $21.9 \%$ (41), $\mathrm{p}=0.015$, respectively). It was indicated the suppression of the proportion of "classic" NS (54.6\% (102), p=0.028) and the group of patients with isolated hematuria (18.2\% (34), $\mathrm{p}=0.024)$. Besides the higher frequency of the initial renal insufficiency was observed after the year $2000(51.3 \%$ (96) vs $27.7 \%$ (322), $\mathrm{p}<0.001)$.

Histological examinations of all 139 biopsied SR children revealed the following distribution: $18.7 \%$ (26) had Focal Segmental Glomerulosclerosis (FSGS), 9.4\% (13) had Minimal Change Disease (MCD), and 39.5\% (55) had Diffuse Mesangial Proliferation (DMP). The remaining patients were classified as Membranoproliferative or Fibroplastic glomerulonephritis, 18.0\% (25) and 14.4\% (20), respectively.

Prevalence of DMP was discovered during the years 1980-2000 unlike the second time interval, when almost one half of biopsies revealed MCD or FSGS which is probably due to the diagnostic uncertainties (Table 2). The proportion of patients with Membranoproliferative glomerulonephritis (all cases diagnosed as type I) decreased in the years 20012006. No case of Membranous glomerulonephritis was found during the whole observational time. Fibroplastic glomerulonephritis, i.e. global sclerosis in the majority of glomerulus, combined with fibroplastic and atrophic changes in interstitium, was seen in each $6-7^{\text {th }}$ kidney biopsy as the dramatic result of other histological damages.

A total of $52.1 \%$ (703) children from the whole cohort responded to the initial PRED therapy, whereas the remaining 47.9\% (646) had SR NS. The age distribution of steroid sensitivity was changed over the time intervals observed (Fig. 2). The percentage of SS NS children decreased in both the youngest and 9-12 years old groups. This percentage was stably high in patients aged from three to six years old and stably low in the oldest age group, clearly indicating the absence of any significant changes in PRED sensitivity among the two time periods. The only group in which the proportion of SS NS cases increased in 

11

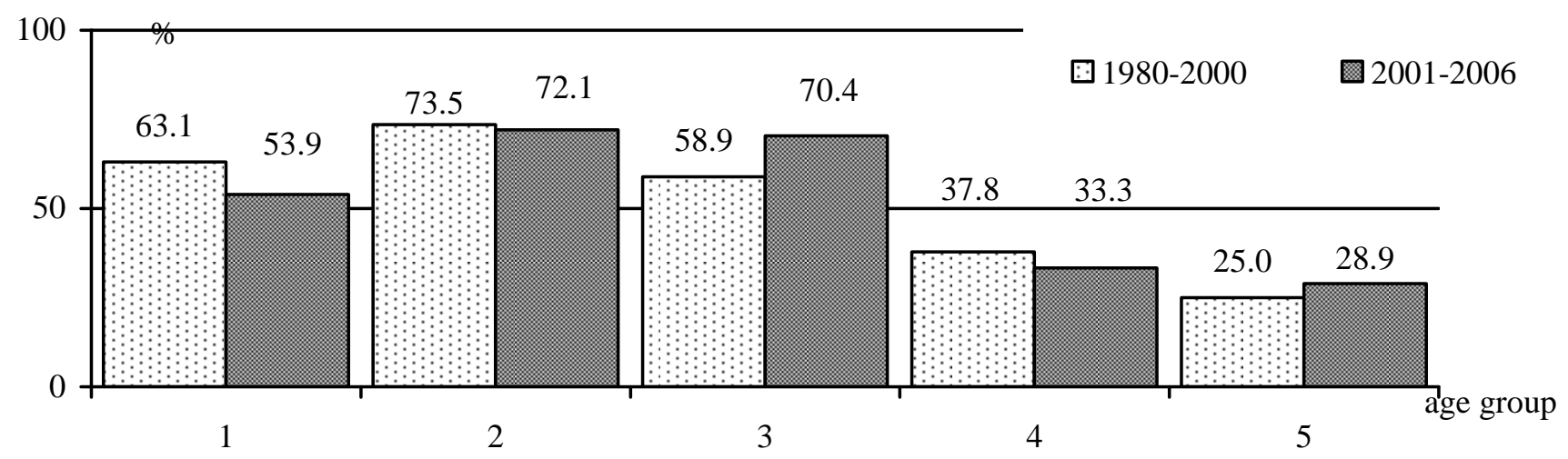

Fig. (2). The response to PRED (first episode): age spectrum of childhood SS NS (PRED prednisolone, SS steroid sensitive, NS nephrotic syndrome).

the years 2001-2006 was the age group from six to nine years.

Table 2. Histopathology Reported in Childhood SR NS (n/\%) (SR Steroid Resistance, NS Nephrotic Syndrome, $M C D$ Minimal Change Disease, DMP Diffuse Mesangial Proliferation, FSGS Focal Segmental Glomerulosclerosis)

\begin{tabular}{|l|c|c|c|}
\hline \multirow{2}{*}{\multicolumn{1}{|c|}{ Glomerular Lesions }} & \multicolumn{2}{c|}{ Time Period } & \multirow{2}{*}{ p-Value } \\
\cline { 2 - 4 } & $\mathbf{1 9 8 0 - 2 0 0 0}$ & $\mathbf{2 0 0 1 - 2 0 0 8}$ & \\
\hline \hline MCD & $7 / 6.9$ & $6 / 15.8$ & 0.073 \\
\hline DMP & $44 / 43.6$ & $11 / 29.0$ & $\mathbf{0 . 0 4 6}$ \\
\hline FSGS & $15 / 14.9$ & $11 / 29.0$ & $\mathbf{0 . 0 3 4}$ \\
\hline $\begin{array}{l}\text { Membranoproliferative } \\
\text { glomerulonephritis }\end{array}$ & $21 / 20.8$ & $4 / 10.4$ & 0.078 \\
\hline Fibroplastic glomerulonephritis & $14 / 13.9$ & $6 / 15.8$ & 0.200 \\
\hline Total number of biopsies & 101 & 38 & \\
\hline
\end{tabular}

Analysis of the clinical presentations dynamics exhibited an increase in the percentage of SR cases among "classic" NS (from 20.1\% (139) to $26.5 \%$ (102), $p=0.035$ ), and a decrease of these cases among NS with hematuria and/or hypertension (from $87.0 \%$ (410) to $80.5 \%$ (68), $\mathrm{p}=0.039$ ).

The CKD3 rate is one of the crucial characteristics of the NS treatment effectiveness. A significant decrease in the percentage of CKD3 outcomes was found in the entire cohort over the years 2001-2006 in contrast to the years $1980-2000$ (11.2\% (21) vs $17.6 \%$ (204), $\mathrm{p}=0.008)$. The fatal ESRD was present in $14.5 \%$ (168) in the first period of study, and $6.4 \%$ (12) in the second time period.

The differences in the treatment regimens were observed in pre-specified time intervals. PRED-monotherapy prescribed in 29.2\% (339) and 24.1\% (45) in 1980-2000 and 2001-2006, respectively. Since 2000, the protocol with pulse Methylprednisolone was used for some patients $(11.8 \%$ (23)). Up to the year 2001, the Chlorambucil treatment was applied in 51.2\% (595), being reduced in 2001-2006, 25.1\% (47). The use of Cyclophosphamide I.V. was rare up to 2001 showing 4.4\% (51), and Cyclophosphamide I.M. mode was preferred in $15.2 \%$ (177). In 1980-1990, the I.M. method was mainly used in patients who did not respond to the Chlorambucil pretreatment or in the case of severe edema. The same treatment was also induced in teenagers due to unfavorable prognostic patterns in this age group. After the year 2000 we used more saving programs: only a few patients, 3.7\% (7), received Cyclophosphamide I.M., whereas $32.1 \%$ (60) was treated by I.V. mode. Additionally, the selective immunosuppressive agents were initiated as a second line treatment. Cyclosporine was prescribed in the dose 5-6 mg/kg/day (whole blood level 90-120 ng/ml) for 12 months in $5.9 \%$ (8). The Mofetyl Mycophenolate treatment had the same duration with doses $500-600 \mathrm{mg} / \mathrm{m}^{2} /$ day in $9.1 \%$ (17) [18]. Both therapy programs were combined with PRED supporting dose during the first 6-9 months.

To be more specific in analyzing the treatment effectiveness, we first focused on the clinical outcomes of SS NS in two time periods. $34.7 \%$ (213) and 33.4\% (30) of SS children relapsed in the first and second time periods, respectively $(\mathrm{p}=0.092)$. Moreover, of those initially SS NS $1.8 \%$ (11) became secondary SR in the period 1980-2000, and the percentage of these patients increased significantly up to $5.6 \%$ (5) during the later time period $(\mathrm{p}=0.032)$.

We then analyzed the outcomes of SR NS where the remission was observed in more than half of the cases (Fig. 3). The therapy response covering both complete and partial remission, varied between $52 \%$ and $57 \%$ in the time periods. It is important to notice that the complete remission was seen more often before the year 2001. Furthermore, both complete and partial remission were more stable, i.e. gave significantly less relapse in the earlier time interval (for complete permission $15.8 \%$ vs $35.0 \%, \mathrm{p}=0.030$; and for partial permission $16.1 \%$ vs $22.6 \%$, $\mathrm{p}=0.122$ ).

At the same time, the percentage of non-remission SR patients was considerably higher after the year 2000; (see Fig. 3). At the end of the follow up period, these patients did not demonstrate any features of CKD3, being still classified as the NS status. Even though the average time of developing CKD3 was estimated to be $14 \pm 23$ months and $13 \pm 13$ months, respectively, one cannot exclude the possibility of CKD3 in those non-remission SR children which were followed up more than 36 but less than 60 months $(9.3 \%$ (17) in the years $1980-2000$ and $16.2 \%$ (7) in the years 2001-2006, $\mathrm{p}=0.045)$. 


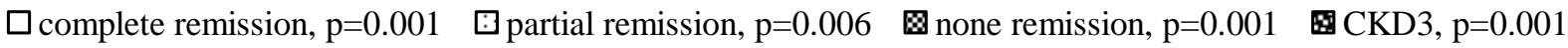

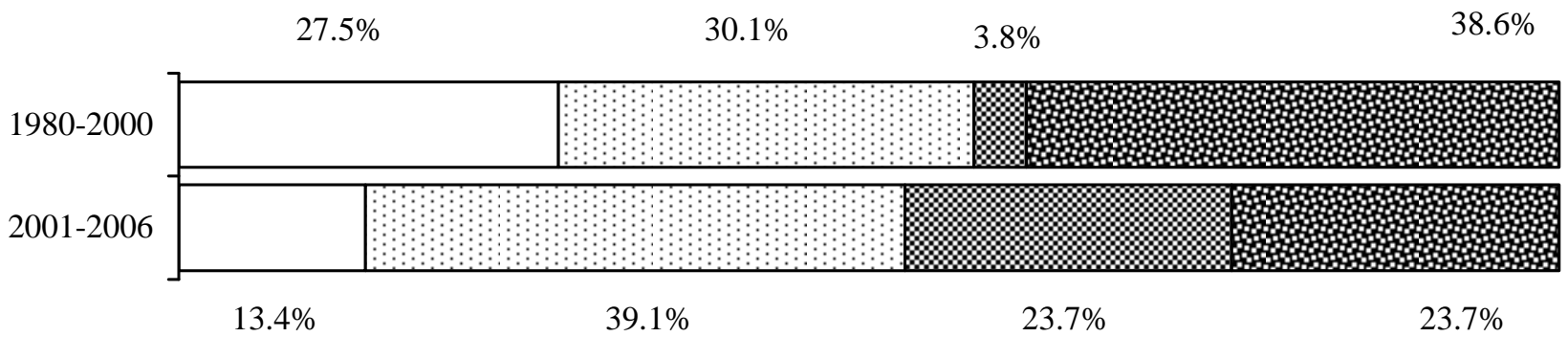

Fig. (3). Outcomes of SR NS in children ( $R R$ steroid resistance, $N S$ nephrotic syndrome, $C K D 3$ chronic kidney disease, stage 3 ).

To evaluate the time dynamics of the risk of developing CKD3 among SR NS during a five years period since the beginning of observation in the clinic, we exploited the Kaplan-Meier survival function technique and estimated the probability (or risk) of CKD3 in the two periods under study. The Kaplan-Meier graphs represented the growth of the cumulative probability of developing CKD3 corrected by the censored times, i.e. by taking into account that the period of observation for some patients was cut off before the event of interest occurred (Fig. 4). The resulting estimates of fiveyear risk of developing CKD3 were $39.7 \%$ for SR patients admitted between the years 1980 and 2000 , and $27.7 \%$ for the patients admitted between the years 2001 and 2006, respectively. The graphs indicated a clear shift between the two cumulative probability functions and the significant difference in the risk of CKD3 for the two time intervals was found using a long-rank test (log-rank $=2.67, p=0.008)[16]$.

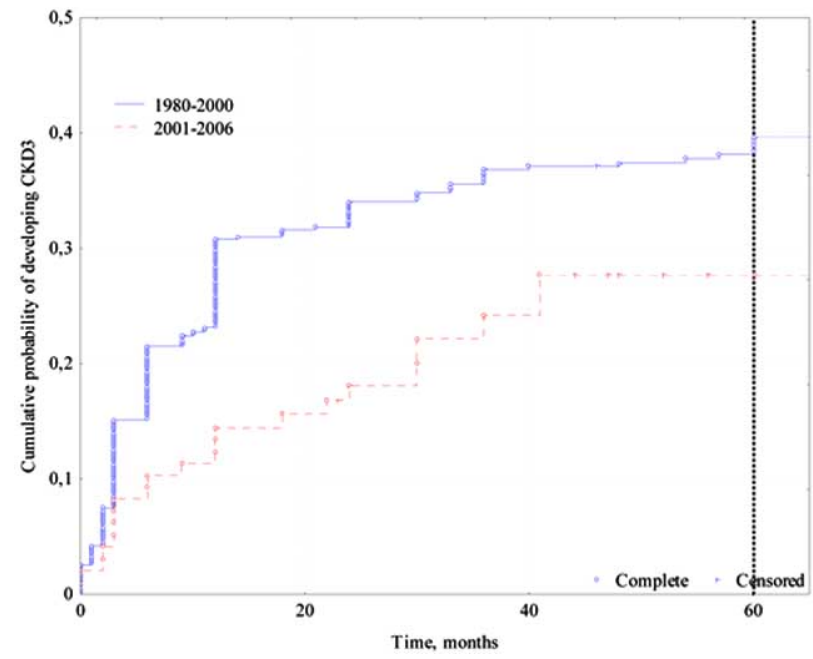

Fig. (4). Kaplan-Meyer analysis of the risk of CKD3 in childhood SR NS: total number - 646, complete - $225(34.8 \%)$, censored: 421 (65.2\%) (CKD3 chronic kidney disease, stage 3, NS nephrotic syndrome, $S R$ steroid resistance).

To capture possible predictive factors of $\mathrm{CKD} 3$ in nephrotic children, the Cox regression model was fitted to the data observed over two time periods (Table 3). The response variable in the Cox regression expressed the hazard or risk of developing CKD3 at each small time interval over the follow-up as a function of several explanatory variables, which are known to affect the probability of CKD3 outcome and cannot be easily controlled for during the treatment. See the more detailed presentation of the actual method of the Cox regression modeling in $[16,17]$.

The four variables, sex, age group, hypertension and the initial renal insufficiency, appeared to be highly significant individual prognostic factors for the risk of CKD3 in SR NS in the years 1980-2000 (Table 3). Observe however, that the univariate approach relied on the independence between the feature variables, thereby totally ignoring the fact that the predictive potential of a variable may change when other variables also are included in the model. To simultaneously explore predictive properties of several variables on the risk of CKD3, we complemented our analysis by the multivariate Cox regression technique which partly confirmed our findings form the univariate analysis. Both initial renal insufficiency and hypertension remained highly significant predictive potential in the multivariate Cox model in the years 1980-2000 (Table 3). No individually significant predictors of CKD3 as found in the years 2001-2006, and therefore we did not proceed with fitting a multivariate Cox regression model.

The estimated HR was reported in the Table $\mathbf{3}$ for each risk predictor variable, together with their $95 \% \mathrm{CI}$. They allows for evaluation of the proportional change that can be expected in the hazard of developing $\mathrm{CKD} 3$, related to changes in the explanatory variables. For example, we found that the risk of CKD3 among girls with SR NS before the year 2001 was $32 \%$ higher than for boys $(p=0.046)$. However, during the later time period, only a tendency of the risk decrease was observed, giving $22 \%$ higher risk for boys. A grows of estimated HR in various age groups was observed over two time periods. In the years 1980-2000, the smallest HR was observed in the age group 2, indicating the lowest risk of developing CKD3. However, during the later time period, this age group demonstrated the most unfavorable pattern, with an HR of 1.8 (Table 3). The clinical risk related variables, such as hematuria, hypertension, and initial renal failure, have shown relatively stable point estimates of $\mathrm{HR}$, however not achieving required significance in the years 2001-2006 which could be due to small sample size in this time periods. Observe that the CI was longer in the second time period, reflecting higher estimator's uncertainty, which could also be interpreted as a small sample effect. 
Table 3. Risk Factors for CKD3 in Childhood SR NS (CKD3 Chronic Kidney Disease, Stage 3, NS Nephrotic Syndrome, SR Steroid Resistance, HR Hazard Ratio, CI Confidence Interval)

\begin{tabular}{|c|c|c|c|c|c|c|c|c|}
\hline \multirow{3}{*}{ Characteristic } & \multicolumn{8}{|c|}{ Time Period } \\
\hline & \multicolumn{2}{|c|}{ Univariate } & \multicolumn{2}{|c|}{ Multivariate } & \multicolumn{2}{|c|}{ Univariate } & \multicolumn{2}{|c|}{ Multivariate } \\
\hline & HR (95\% CI) & p-Value & HR (95\% CI) & p-Value & HR $(95 \%$ CI) & p-Value & HR $(95 \%$ CI) & p-Value \\
\hline Female & $1.32(1.01-1.74)$ & 0,046 & & & $0.78(0.33-1.84)$ & 0.570 & & \\
\hline 1 & 1 & & & & 1 & & & \\
\hline 2 & $0.42(0.24-0.76)$ & 0.004 & & & $1.80(0.48-6.76)$ & 0.380 & & \\
\hline 3 & $0.57(0.34-0.94)$ & 0.029 & & & $0.91(0.16-5.05)$ & 0.910 & & \\
\hline 4 & $0.73(0.45-1.18)$ & 0.200 & & & $0.60(0.16-2.25)$ & 0.450 & & \\
\hline 5 & $0.68(0.43-1.09)$ & 0.110 & & & $0.41(0.11-1.53)$ & 0.180 & & \\
\hline Initial renal insufficiency & $2.62(1.97-3.48)$ & $<0.001$ & $2.20(1.63-2.98)$ & $<0.001$ & $2.09(0.70-6.23)$ & 0.180 & & \\
\hline
\end{tabular}

\section{DISCUSSION}

This is the single largest retrospective study performed in Ukraine comparing the demographic profiles and clinical outcome in children with NS between two diverse timeperiods. The focus of this study was on the selective cohort representing the most complicated NS cases.

The analysis of the demographic data showed that the median age of our patients at the onset of NS did not change between the two time periods, and was estimated to be 6.7 and 6.8 years, respectively, unlike the internationally reported data that assigned the average peaking of "classic" NS to 3-4 years $[2,3,6]$. These discrepancies might be explained by the selective properties of our cohort.

Changes in the age distribution and clinical patterns were found. A significant decrease the proportion of the age group of children, traditionally sensitive to the therapy (from three to nine years old), in combination with higher levels of atypical NS due to concomitant hypertension and initial renal impairment in 2001-2006 was observed. Unlike international data [1-6], our results also revealed a higher proportion of patients with SR for both time periods. These findings were the result of patient selection partly. An alternate explanation could be related to substantial changes in the NS pattern, thereby supporting the hypothesis on evolutionary processes in the disease course.

One of the important histological findings of our study was a growth of various types of FSGS in SR NS patients observed between the two time periods. These results were also supported by international reports [1, 2, 8, 19-22]. The percentage of SR patients with DMP appeared to be far higher than in other studies $[1,3,10]$ and could partly be explained by our laboratorial limitations before the year 2004. A stably high level of Fibroplastic Glomerulonephritis was found, indicating that the late biopsy testing was relatively common in both time periods, and that the clinical picture was more "aggressive". Observe however that our histological conclusions were not free from bias induced by a small sample of biopsy tests analyzed, in combination with the selective cohort properties.

The well-established international treatments strategy for NS children suggested PRED as the first-line therapy [2325]. In general, childhood SSNS resulted in a complete remission, exhibiting however subsequent relapse in 50 to $80 \%$ of the cases $[5,9,26]$. Our practice of administration of the cytostatic agent directly after the first relapse significantly reduced the number of subsequent relapse rate, appearing to be about $33 \%$ for both time-periods. These results were achieved even though part of the patients with less than three years follow-up was excluded from study. Given that the losses in follow up might be explained by no relapsing, we could have had even more positive results in reducing the relapse rate by our treatment strategy.

The secondary SR (i.e. SR after relapse) is one of the features of poor prognosis. We found increasing SR in 20012006 years among initially SS patients $(5.6 \%$ vs $1.8 \%$ in the first period of study). These findings contributed to the negative trends discovered in the NS evolution especially in comparison to the previously reported results where only 1 to $3 \%$ of the relapsing nephrotic children developed the secondary SR $[4,9]$.

While the clinical management of SSNS seems to be relatively straightforward, the choice of the optimal treatment strategy of SRNS essentially depends on the histological patterns and generally was much more unclear. As already mentioned the principal differences between our and the international approaches to the treatment of NS is existed based on socio-economic conditions in Ukraine and relatively limited possibilities to the kidney replacement therapy. In order to provide a safety treatment we administrate both the prolonged initial PRED and higher cumulative immunosuppressant's doses. Our earlier studies have demonstrated their effectiveness in retarding the progression of renal damage $[11,15]$. In spite of unavoidable 
side-effects reported in international studies [23, 24, 27], the "aggressive" therapy can be used in nephrotic children in Ukraine since it improves or at least maintains their ongoing life quality. The detailed analysis of side effects and dramatic complications from the prolonged treatment was beyond the scope of this study. Despite the differences, the effectiveness of domestic treatment regimes was relatively similar to international results. The rate of CKD3 in our cohort was $17.6 \%$ in the years $1980-2000$ and $11.2 \%$ for second time period which agreed with the international data reporting a renal survival rate of about $65-92 \%[3,5,19,20$, $23,28-30]$. In addition it is worth noting that some reviews have also recommended the prolonged courses of both PRED and cytostatic agents $[1,20,25,26]$.

The earlier treatment strategy, while being more "aggressive", demonstrated better result in achieving complete remission. At the same time this strategy led to a higher frequency of developing CKD3 and gave no efficient retard of the decline of renal function in the absence of remission, unlike the treatment approaches accepted in the years 2001-2006. Some uncertainty in these conclusions was related to the study limitations discussed above, namely, that a follow-up time for part of the patients was shorter than 60 months. To overcome this limitation, we analyzed the cumulative probability of development CKD3 using the Kaplan-Meier survival estimation technique, a method which made it possible to correct probability estimations for different follow-up times. A significant decrease in the risk of CKD3 in the years 2001-2006 was observed. Improvement in the effectiveness of therapy was the result of changes in NS treatment strategy despite the increase in the proportion of patients with atypical course of disease in the last time interval.

The most important risk factor of the unfavorable prognosis of NS was the unresponsiveness to PRED. The interest in the identifying predictive factors for developing CKD3 was shown in many studies [3, 19, 20, 22, 27-31]. Generally, internationally reported results were essentially heterogenic and uncertain being usually obtained on much smaller samples than those in our study. We found that gender, age, hypertension and initial renal insufficiency (each individually) was significant in the prediction CKD3 for the first period of study, and last two of these variables remained highly significant even in the multivariate Cox model. However, none of them were significant in the years 2001-2006, which could be due to much smaller sample size as well as to shorter follow up period. Unless though statistical significance was not achieved, some of these variables, such as hypertension and initial renal insufficiency as well as their combination, could have high clinical significance. Nevertheless, the tendency observed in 20012006 is towards a higher hazard of developing CKD3 in male patients in the age from three to six years. Such combination has never been reported early and could contribute to generating hypotheses in searching for informative CKD3 predictors.

\section{CONCLUSION}

Our results suggest that a qualitative change in the course of childhood NS occurred between the years 1980-2000 and 2001-2006 revealing more severe patterns of the disease in the second time period. This supports the hypothesis about the evolutionary processes in the disease and indicates the need for updating of the current international NS management. The motivation for consideration of Ukrainian treatment strategy is the effectiveness of prolonged courses of PRED and immunosuppressive agents in reducing the relapse rate in SSNS and in retarding the progression of renal damage in SRNS.

\section{ACKNOWLEDGEMENTS}

The authors wish to thank Dr. V. Nepomnyashchy for providing histopathological data review. The study was in part supported by the grant N00962/2008 from the Visby Program, Swedish Institute (http://www.si.se).

\section{REFERENCES}

[1] Wong W. Idiopathic nephrotic syndrome in New Zealand children, demographic, clinical features, initial management and outcome after twelve-month follow-up: results of a three-year national surveillance study. J Paediatr Child Health 2007; 43: 337-41.

[2] Kim JS, Bellew CA, Silverstein DM, Aviles DH, Boineau FG, Vehaskari VM. High incidence of initial and late steroid resistance in childhood nephrotic syndrome. Kidney Int 2005; 68: 1275-81.

[3] Mekahli D, Liutkus A, Ranchin B, et al. Long-term outcome of idiopathic steroid-resistant nephrotic syndrome: a multicenter study. Pediatr Nephrol 2009; 24: 1525-32.

[4] Niaudet P. Lipoid nephrosis in childhood [in French]. Rev Prat 2003; 53: 2027-32.

[5] Avner ED, Harmon WE, Niaudet P, Eds. Pediatric nephrology. 5th edn. Philadelphia: Lippincott Williams \& Wilkins 2004; 543-73.

[6] McKinney PA, Feltbower RG, Brocklebank JT, Fitzpatrick MM. Time trends and ethnic patterns of childhood nephrotic syndrome in Yorkshire, UK. Pediatr Nephrol 2001; 16: 1040-4.

[7] Eddy AA, Symons JM. Nephrotic syndrome in childhood. Lancet 2003; 362: 629-39.

[8] International Study of Kidney Disease in Children. The primary nephrotic syndrome in children. Identification of patients with minimal change nephrotic syndrome from initial response to prednisolone. A report of the International Study of Kidney Disease in Children. J Pediatr 1981; 98: 561-4.

[9] Tarshish P, Tobin JN, Bernstein J, Edelmann CM. Prognostic significance of the early course of minimal change nephrotic syndrome: report of the International Study of Kidney Disease in Children. J Am Soc Nephrol 1997; 8: 769-76.

[10] Tune BM, Mendoza SA. Treatment of idiopathic nephrotic syndrome: regimens and outcomes in children and adults. J Am Soc Nephrol 1997; 8(5): 824-32.

[11] Bagdasarova I, Fomina S. Diagnosis and treatment of nephrotic syndrome in children [in Russian]. Cur Pediatr (UA) 2005; 6: 1548.

[12] Rees L, Webb NJA, Brogan PA, Eds. Pediatric nephrology. Oxford: University Press 2007; 13-21: 228-245.

[13] Schwartz GJ, Brion LP, Spitzer A. The use of plasma creatinine concentration for estimating glomerular filtration rate in infants, children, and adolescents. Pediatr Clin North Am 1987; 34: 571-90.

[14] K/DOQI. Clinical practice guidelines for chronic kidney disease: evaluation, classification and stratification. Am J Kidney Dis 2002; 39: S1-S266.

[15] Fomina S, Bagdasarova I. Outcomes of glucocorticoid therapy in the children with steroid sensitive and steroid resistance nephrotic syndrome. WCN2009 Su754 [serial on the internet]. 2009 May; [cited 2009 May 24]. Available from: http://www.postersession online.com/173580348_eu/congresos/WCN2009/aula/poster_2448 7.pdf

[16] Altman DG. Practical statistics for medical research. $1^{\text {st }}$ ed. London: Chapman \& Hal 1991; 543-73.

[17] Rice JA. Mathematical Statistics and Data Analysis. $2^{\text {nd }}$ ed. Duxbury Press 2006; 348-53.

[18] Fomina S, Bagdasarova I. Efficiency of Cyclosporin A and Mycophenolate Mofetil in the children with steroid sensitive nephrotic syndrome. WCN2009 Su732 [serial on the internet]. 2009 May; [cited 2009 May 24]. Available from: 
http://www.postersessiononline.com/173580348_eu/congresos/WC N2009/aula/-Sun_732_WCN2009.pdf

[19] Gipson DS, Chin H, Presler TP, et al. Differential risk of remission and ESRD in childhood FSGS. Pediatr Nephrol 2006; 21: 344-9.

[20] Paik KH, Lee BH, Cho HY, et al. Primary focal segmental glomerular sclerosis in children: clinical course and prognosis. Pediatr Nephrol 2007; 22: 389-95.

[21] Chesney R. The changing face of childhood nephrotic syndrome. Kidney Int 2004; 66: 1294-302.

[22] Borges FF, Shiraichi L, da Silva MP, Nishimoto EI, Nogueira PC. Is focal segmental glomerulosclerosis increasing in patients with nephrotic syndrome? Pediatr Nephrol 2007; 22: 1309-13.

[23] Hodson EM, Habashy D, Craig JC. Interventions for idiopathic steroid-resistant nephrotic syndrome in children. 2006 Cochrane Database Syst Rev (2):CD003594. DOI: 10.1002/14651858.CD00 3594.pub3 [updated 2008, cited 2007 Nov 7]. Available from: http://www.thecochranelibrary.com/

[24] Hodson EM, Willis NS, Craig JC. Non-corticosteroid treatment for nephrotic syndrome in children. Cochrane Database Syst Rev (1):CD002290. DOI: 10.1002/14651858.CD002290.pub3 [updated 2008, cited 2006 Jan 9]. Available from: http://www.thecochrane library.com/
[25] Hiraoka M, Tsukahara H, Matsubara K, et al. A randomized study of two long-course prednisolone regimens for nephrotic syndrome in children. Am J Kid Dis 2003; 41(6): 1155-62.

[26] Letavernier B, Letavernier E, Leroy S, Baudet-Bonneville V, Bensman A, Ulinski T. Prediction of high-degree steroid dependency in pediatric idiopathic nephrotic syndrome. Pediatr Nephrol 2008; 23: 2221-6.

[27] Abeyagunawardena AS, Sebire NJ, Risdon RA, et al. Predictors of long-term outcome of children with idiopathic focal segmental glomerulosclerosis. Pediatr Nephrol 2007; 22: 215-21.

[28] Martinelli R, Okumura AS, Pereira LJ, Rocha H. Primary focal segmental glomerulosclerosis in children: prognostic factors. Pediatr Nephrol 2001; 16: 658-61.

[29] Abrantes MM, Cardoso LSB, Lima EM, et al. Predictive factors of chronic kidney disease in primary focal segmental glomerulosclerosis. Pediatr Nephrol 2006; 21: 1003-12.

[30] Ehrich JHH, Geerlings C, Zivicnjak M, Franke D, Geerlings H, Gellermann J. Steroid-resistant idiopathic childhood nephrosis: overdiagnosed and undertreated. Nephrol Dial Transplant 2007; 22: 2183-93.

[31] Coppo R. Non-steroidal and non-cytotoxic therapies for nephrotic syndrome. Nephrol Dial Transplant 2008; 23: 1793-6.

(C) Fomina et al.; Licensee Bentham Open.

This is an open access article licensed under the terms of the Creative Commons Attribution Non-Commercial License (http://creativecommons.org/licenses/by$\mathrm{nc} / 3.0 /$ ) which permits unrestricted, non-commercial use, distribution and reproduction in any medium, provided the work is properly cited. 